\title{
$\mathbf{B}_{\mathrm{s}}^{0}$ Decays at Belle
}

\section{Remi Louvot*}

(On behalf of the Belle collaboration)

Laboratoire de Physique des Hautes Énergies,

École Polytechnique Fédérale de Lausanne (EPFL), Lausanne, Switzerland

E-mail: remi.louvoteepfl.ch

The large data sample being recorded with the Belle detector at the $\Upsilon(5 S)$ energy provides a unique opportunity to study the poorly-known $B_{s}^{0}$ meson. Three analyses, using a sample of $23.6 \mathrm{fb}^{-1}$, are presented. We report the first observation of the three dominant exclusive $B_{s}^{0}$ decays, $B_{s}^{0} \rightarrow D_{s}^{*-} \pi^{+}, B_{s}^{0} \rightarrow D_{s}^{-} \rho^{+}$and $B_{s}^{0} \rightarrow D_{s}^{*-} \rho^{+}$, the first observation of the $C P$-eigenstate decay $B_{s}^{0} \rightarrow J / \psi \eta$, and the results from a study of the charmless $B_{s}^{0} \rightarrow K^{+} K^{-}, B_{s}^{0} \rightarrow K^{+} \pi^{-}$, $B_{s}^{0} \rightarrow \pi^{+} \pi^{-}$and $B_{s}^{0} \rightarrow K_{S}^{0} K_{S}^{0}$ decays. The following branching fraction measurements are presented: (When mentioned, the systematic errors due to the uncertainties on the fraction $f_{s}=$ $\sigma\left(e^{+} e^{-} \rightarrow B_{s}^{(*)} \bar{B}_{s}^{(*)}\right) / \sigma_{b \bar{b}}$ and on the decay polarization (pol.) are quoted separately.)

$$
\begin{gathered}
\left.\left.\mathscr{B}\left(B_{s}^{0} \rightarrow D_{s}^{*-} \pi^{+}\right)=\left(2.4_{-0.4}^{+0.5} \text { (stat. }\right) \pm 0.3 \text { (syst. }\right) \pm 0.4\left(f_{s}\right)\right) \times 10^{-3}, \\
\left.\left.\mathscr{B}\left(B_{s}^{0} \rightarrow D_{s}^{-} \rho^{+}\right)=\left(8.5_{-1.2}^{+1.3} \text { (stat. }\right) \pm 1.1 \text { (syst. }\right) \pm 1.3\left(f_{s}\right)\right) \times 10^{-3}, \\
\left.\left.\mathscr{B}\left(B_{s}^{0} \rightarrow D_{s}^{*-} \rho^{+}\right)=\left(13.0_{-2.1}^{+2.3} \text { (stat. }\right) \pm 1.7 \text { (syst. }\right) \pm 1.7(\text { pol. }) \pm 1.9\left(f_{s}\right)\right) \times 10^{-3}, \\
\left.\left.\mathscr{B}\left(B_{s}^{0} \rightarrow J / \psi \eta\right)=(3.69 \pm 0.95 \text { (stat. })_{-0.95}^{+0.65} \text { (syst. }\right)\right) \times 10^{-4} \text { and } \\
\left.\left.\mathscr{B}\left(B_{s}^{0} \rightarrow K^{+} K^{-}\right)=\left(3.8_{-0.9}^{+1.0} \text { (stat. }\right) \pm 0.7 \text { (syst. }\right)\right) \times 10^{-5},
\end{gathered}
$$

and the limits at $90 \%$ confidence level:

$$
\begin{gathered}
\mathscr{B}\left(B_{s}^{0} \rightarrow \pi^{+} \pi^{-}\right)<1.2 \times 10^{-5}, \\
\mathscr{B}\left(B_{s}^{0} \rightarrow K^{+} \pi^{-}\right)<2.6 \times 10^{-5} \text { and } \\
\mathscr{B}\left(B_{s}^{0} \rightarrow K^{0} \bar{K}^{0}\right)<6.6 \times 10^{-5} .
\end{gathered}
$$

December 15, 2009

The 2009 Europhysics Conference on High Energy Physics,

July 16 - 22, 2009

Krakow, Poland

${ }^{*}$ Speaker. 


\section{Introduction}

The Belle experiment [1], located at the interaction point of the KEKB asymmetric-energy $e^{+} e^{-}$collider [2], was designed for the study of $B$ mesons ${ }^{1}$ produced in $e^{+} e^{-}$annihilation at a center-of-mass $(\mathrm{CM})$ energy corresponding to the mass of the $\Upsilon(4 S)$ resonance $(\sqrt{s} \approx 10.58 \mathrm{GeV})$. After having recorded an unprecedented sample of $\sim 800$ millions of $B \bar{B}$ pairs, the Belle collaboration started to record collisions at higher energies, opening the possibility to study other particles, like the $B_{s}^{0}$ meson. Up to now, $L_{\text {int }}=23.6 \mathrm{fb}^{-1}$ of data have been analyzed at the energy of the $\Upsilon(5 S)$ resonance $(\sqrt{s} \approx 10.87 \mathrm{GeV})$.

The $\Upsilon(5 S)$ resonance is above the $B_{s}^{0} \bar{B}_{s}^{0}$ threshold and it was naturally expected that the $B_{s}^{0}$ meson could be studied with $\Upsilon(5 S)$ data as well as the $B$ mesons are with $\Upsilon(4 S)$ data. The large potential of such $\Upsilon(5 S)$ data was quickly confirmed [3, 4] with the 2005 engineering run representing $1.86 \mathrm{fb}^{-1}$. The main advantage with respect to the hadronic colliders is the possibility of measurements of absolute branching fractions. However, the abundance of $B_{s}^{0}$ mesons in $\Upsilon(5 S)$ hadronic events has to be precisely determined. Above the $e^{+} e^{-} \rightarrow u \bar{u}, d \bar{d}, s \bar{s}, c \bar{c}$ continuum events, the $e^{+} e^{-} \rightarrow b \bar{b}$ process can produce different kinds of final states: seven with a pair of non-strange $B$ mesons $\left(B^{*} \bar{B}^{*}, B^{*} \bar{B}, B \bar{B}, B^{*} \bar{B}^{*} \pi, B^{*} \bar{B} \pi, B \bar{B} \pi\right.$ and $\left.B \bar{B} \pi \pi\right)$, three with a pair of $B_{s}^{0}$ mesons $\left(B_{s}^{*} \bar{B}_{s}^{*}\right.$, $B_{s}^{*} \overline{B_{s}^{0}}$ and $B_{s}^{0} \overline{B_{s}^{0}}$ ), and final states involving a bottomonium resonance below the $B \bar{B}$ threshold [5]. The $B^{*}$ and $B_{s}^{*}$ mesons always decay by emission of a photon. The total $e^{+} e^{-} \rightarrow b \bar{b}$ cross section at the $\Upsilon(5 S)$ energy was measured to be $\sigma_{b \bar{b}}=(302 \pm 14) \mathrm{pb}[3,6]$ and the fraction of $B_{s}^{0}$ events to be $f_{s}=\sigma\left(e^{+} e^{-} \rightarrow B_{s}^{(*)} \bar{B}_{s}^{(*)}\right) / \sigma_{b \bar{b}}=(19.3 \pm 2.9) \%$ [7]. The dominant $B_{s}^{0}$ production mode, $b \bar{b} \rightarrow$ $B_{s}^{*} \bar{B}_{s}^{*}$, represents $f_{B_{s}^{*} \bar{B}_{s}^{*}}=\left(90.1_{-4.0}^{+3.8} \pm 0.2\right) \%$ of the $b \bar{b} \rightarrow B_{s}^{(*)} \bar{B}_{s}^{(*)}$ events [8].

For all the exclusive modes presented here, the $B_{s}^{0}$ candidates are fully reconstructed from the final-state particles. From the reconstructed four-momentum in the $\mathrm{CM},\left(E_{B_{s}^{0}}^{*}, p_{B_{s}^{0}}^{*}\right)$, two variables are formed: the energy difference $\Delta E=E_{B_{s}^{0}}^{*}-\sqrt{s} / 2$ and the beam-constrained mass $M_{\mathrm{bc}}=$ $\sqrt{s / 4-p_{B_{s}^{0}}^{* 2}}$. The signal coming from the dominant $e^{+} e^{-} \rightarrow B_{s}^{*} \bar{B}_{s}^{*}$ production mode is extracted from a two-dimensional fit performed on the distribution of these two variables. The corresponding branching fraction is then extracted using the total efficiency (including sub-decay branching fractions) determined with Monte-Carlo simulations, $\sum \varepsilon \mathscr{B}$, and the number of $B_{s}^{0}$ mesons produced via the $e^{+} e^{-} \rightarrow B_{s}^{*} \bar{B}_{s}^{*}$ process, $N_{B_{s}^{0}}=2 \times L_{\text {int }} \times \sigma_{b \bar{b}} \times f_{s} \times f_{B_{s}^{*} \bar{B}_{s}^{*}}=(2.48 \pm 0.41) \times 10^{6}$.

\section{Observation of three new CKM-favored $B_{s}^{0}$ decays}

Following our recent measurement of $B_{s}^{0} \rightarrow D_{s}^{-} \pi^{+}$[8], we present for the first time an extension of this analysis which includes decays with photons in the final state and report the first observations of the decays $B_{s}^{0} \rightarrow D_{s}^{*-} \pi^{+}, B_{s}^{0} \rightarrow D_{s}^{-} \rho^{+}$and $B_{s}^{0} \rightarrow D_{s}^{*-} \rho^{+}$. The leading process of these three modes is a $b \rightarrow c \bar{u} d$ tree-level transition of order $\lambda^{2}$ with a spectator $s$ quark. The $D_{s}^{-}$ mesons are reconstructed via three modes : $D_{s}^{-} \rightarrow \phi\left(\rightarrow K^{+} K^{-}\right) \pi^{-}, D_{s}^{-} \rightarrow K^{* 0}\left(\rightarrow K^{+} \pi^{-}\right) K^{-}$and $D_{s}^{-} \rightarrow K_{S}^{0}\left(\rightarrow \pi^{+} \pi^{-}\right) K^{-}$. Based on the ratio of the second and the zeroth Fox-Wolfram moments [9], $R_{2}$, the $e^{+} e^{-} \rightarrow u \bar{u}, d \bar{d}, s \bar{s}, c \bar{c}$ continuum events are efficiently rejected by taking advantage of the difference between their event geometry (jet like, high $R_{2}$ ) and the signal event shape (spherical, low $\left.R_{2}\right)$. The $B_{s}^{0} \rightarrow D_{s}^{*-} \pi^{+}\left(B_{s}^{0} \rightarrow D_{s}^{-} \rho^{+}\right.$and $\left.B_{s}^{0} \rightarrow D_{s}^{*-} \rho^{+}\right)$candidates with $R_{2}$ smaller than

\footnotetext{
${ }^{1}$ The notation " $B$ " refers either to a $B^{0}$ or a $B^{+}$. Moreover, charge-conjugated states are implied everywhere.
} 
$0.5(0.35)$ are kept for further analysis. A best candidate selection, based on the intermediate particle reconstructed masses, is then implemented to keep only one $B_{s}^{0}$ candidate per event. The $M_{\mathrm{bc}}$ and $\Delta E$ distribution of the selected candidates for the three modes are shown in Fig. 1, where the various components of the fit are described.
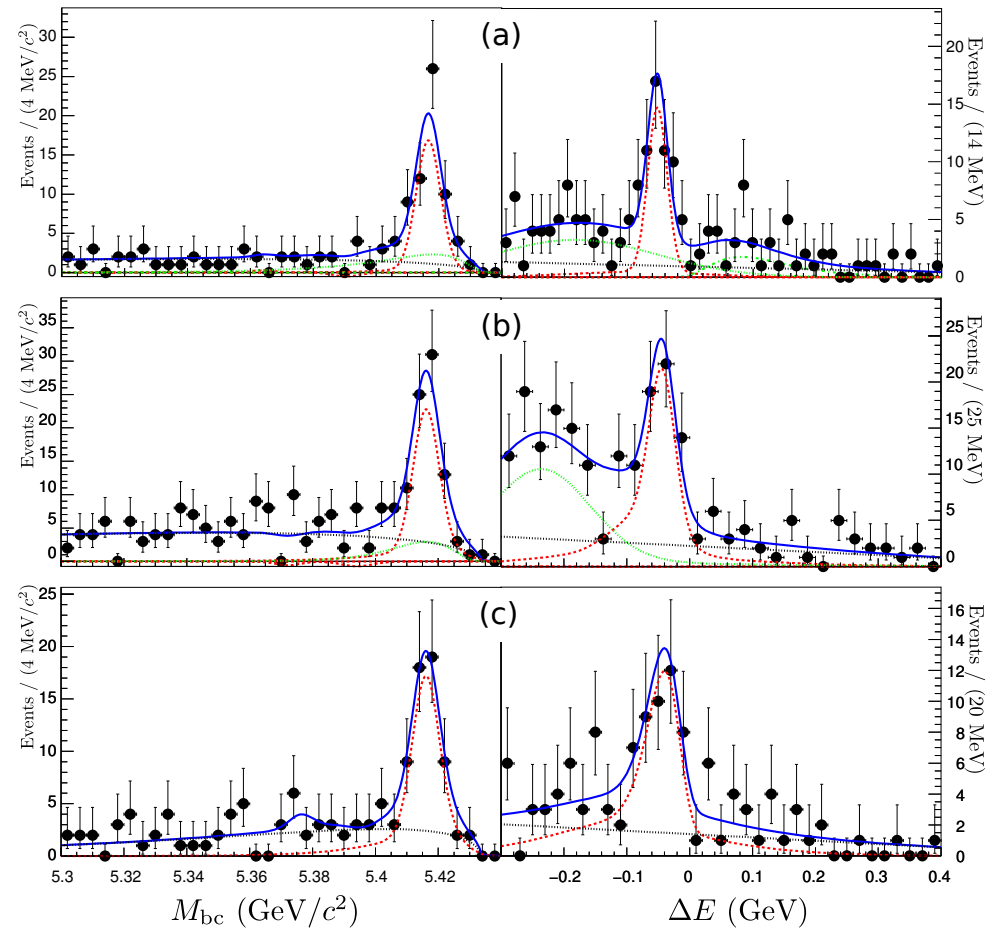

Figure 1: Histograms with the fit results. The left (right) plots shows the $M_{\mathrm{bc}}(\Delta E)$ projections for the candidates with $\Delta E\left(M_{\mathrm{bc}}\right)$ in the $B_{s}^{*} \bar{B}_{s}^{*}$ signal region, The red-dashed (green-dotted, blackdotted, solid-blue) lines represent the signal (peaking $B_{s}^{0}$ background, continuum, total) fitted PDF. (a): $\quad B_{s}^{0} \rightarrow D_{s}^{*-} \pi^{+}$candidates. $B_{s}^{0} \rightarrow D_{s}^{-} \pi^{+}\left(B_{s}^{0} \rightarrow D_{s}^{-} \rho^{+}\right)$ events can be distinguished on the right (left) of the signal peak in the $\Delta E$ plot. (b): $B_{s}^{0} \rightarrow D_{s}^{-} \rho^{+}$candidates. The peaking background comes from $B_{s}^{0} \rightarrow D_{s}^{*-} \rho^{+}$events. (c): $B_{s}^{0} \rightarrow D_{s}^{*-} \rho^{+}$candidates.

The numerical results are summarized in Table 1 . The systematic error coming from $f_{s}$ is separated from the other source of uncertainties. The $B_{s}^{0} \rightarrow D_{s}^{*-} \rho^{+}$mode is the decay of the spinless $B_{s}^{0}$ to two spin-1 particles. The proportion of longitudinal polarization, $f_{L}$, is not known, and the efficiencies depend significantly on this parameter. A $13 \%$ relative error, denoted "pol.", is added to the branching fraction uncertainty to take this into account.

\begin{tabular}{l|ccrc} 
Mode & $N_{\text {sig }}^{B_{s}^{*} \bar{B}_{s}^{*}}$ & $\sum \varepsilon \mathscr{B}\left(10^{-3}\right)$ & $S$ & Branching fraction \\
\hline$B_{s}^{0} \rightarrow D_{s}^{*-} \pi^{+}$ & $53.4_{-9.4}^{+10.3}$ & $9.1 \pm 0.6$ & $8.4 \sigma$ & $\left(2.4_{-0.4}^{+0.5}\right.$ (stat. $) \pm 0.3$ (syst. $\left.) \pm 0.4\left(f_{s}\right)\right) \times 10^{-3}$ \\
$B_{s}^{0} \rightarrow D_{s}^{-} \rho^{+}$ & $92.2_{-13.2}^{+14.2}$ & $4.4 \pm 0.3$ & $10.6 \sigma$ & $\left(8.5_{-1.2}^{+1.3}\right.$ (stat. $) \pm 1.1$ (syst. $\left.\pm 1.3\left(f_{s}\right)\right) \times 10^{-3}$ \\
$B_{s}^{0} \rightarrow D_{s}^{*-} \rho^{+}$ & $86.6_{-14.0}^{+15.1}$ & $2.7 \pm 0.2$ & $8.7 \sigma$ & $\left(13.0_{-2.1}^{+2.3}\right.$ (stat. $) \pm 1.7$ (syst. $) \pm 1.7($ pol. $\left.) \pm 1.9\left(f_{s}\right)\right) \times 10^{-3}$
\end{tabular}

Table 1: Signal yields for the $B_{s}^{*} \bar{B}_{s}^{*}$ production process with statistical error, $N_{\text {sig }}$, total efficiencies, $\sum \varepsilon \mathscr{B}$, statistical significances, $S$, and measured branching fractions for the $B_{s}^{0} \rightarrow D_{s}^{*-} \pi^{+}, B_{s}^{0} \rightarrow D_{s}^{-} \rho^{+}$and $B_{s}^{0} \rightarrow D_{s}^{*-} \rho^{+}$modes.

These first observations confirm the large predominance of the $B_{s}^{*} \bar{B}_{s}^{*}$ production mode as no significant excess is seen in the other two signal regions. Within uncertainties, no deviation from the $S U(3)$-related $B^{0}$ decays is seen.

\section{First observation of the CP-eigenstate decay $\mathbf{B}_{\mathrm{s}}^{\mathbf{0}} \rightarrow \mathrm{J} / \psi \eta$}

$B_{s}^{0}$ decays to $C P$ eigenstates are important for $C P$-violation parameter measurements [10] and 
preliminary results about the first observation of $B_{s}^{0} \rightarrow J / \psi \eta[11]$ are reported. The $J / \psi$ candidates are formed with oppositely-charged electron or muon pairs, while $\eta$ candidates are reconstructed via the $\eta \rightarrow \gamma \gamma$ and $\eta \rightarrow \pi^{+} \pi^{-} \pi^{0}$ modes. A mass (mass and vertex) constrained fit is then applied to the $\eta(J / \psi)$ candidates. If more than one candidate per event satisfies all the selection criteria, the one with the smallest fit residual is selected. The main background is the continuum, which is reduced by requiring $R_{2}<0.4$. The combined $M_{\mathrm{bc}}$ and $\Delta E$ distributions are presented in Fig. 2. The $B_{s}^{0} \rightarrow J / \psi \eta, \eta \rightarrow \gamma \gamma$ and $B_{s}^{0} \rightarrow J / \psi \eta, \eta \rightarrow \pi^{+} \pi^{-} \pi^{0}$ candidates are fitted separately. Table 2 presents the numerical results.
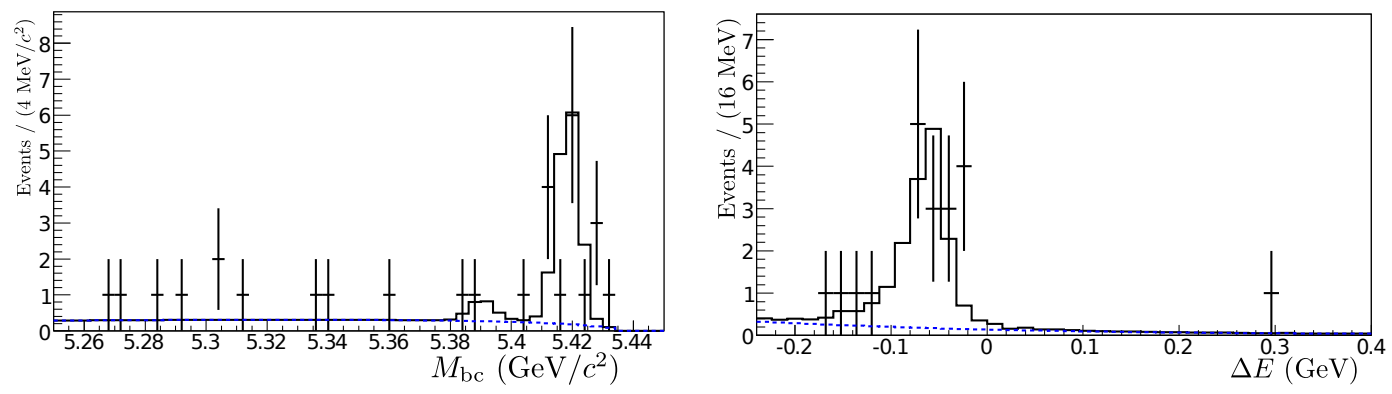

Figure 2: Projection of the $B_{s}^{0} \rightarrow J / \psi \eta$ candidates (points with error bars) and the fitted PDF (solid line) in the $B_{s}^{*} \bar{B}_{s}^{*}$ signal region. The sub-modes $\eta \rightarrow \gamma \gamma$ and $\eta \rightarrow \pi^{+} \pi^{-} \pi^{0}$, which are fitted separately, are summed in these plots. The blue-dotted line represents the continuum component of the PDF. The small peak in the $M_{\mathrm{bc}}$ plot is the $B_{s}^{*} \overline{B_{s}^{0}}$ contribution, as the $B_{s}^{*} \bar{B}_{s}^{*}$ signal range for $\Delta E$ overlaps the one for $B_{s}^{*} \overline{B_{s}^{0}}$ signal.

\begin{tabular}{l|cc} 
& $B_{s}^{0} \rightarrow J / \psi \eta, \eta \rightarrow \gamma \gamma$ & $B_{s}^{0} \rightarrow J / \psi \eta, \eta \rightarrow \pi^{+} \pi^{-} \pi^{0}$ \\
\hline$B_{s}^{*} \bar{B}_{s}^{*}$ Signal Yield & $12.1 \pm 3.8$ & $5.6 \pm 2.5$ \\
Stat. Significance & $5.9 \sigma$ & $4.0 \sigma$ \\
\hline $\mathscr{B}\left(B_{s}^{0} \rightarrow J / \psi \eta\right)$ (combined) & $(3.69 \pm 0.95 \text { (stat. })_{-0.95}^{+0.65}$ (syst. $\left.)\right) \times 10^{-4}$
\end{tabular}

Table 2: $B_{s}^{0} \rightarrow J / \psi \eta$ results: yields, significances and branching fraction.

\section{Observation of $\mathbf{B}_{\mathbf{s}}^{\mathbf{0}} \rightarrow \mathbf{K}^{+} \mathbf{K}^{-}$and searches for $\mathbf{B}_{\mathbf{s}}^{\mathbf{0}} \rightarrow \pi^{+} \pi^{-}, \mathbf{B}_{\mathbf{s}}^{\mathbf{0}} \rightarrow \mathbf{K}^{+} \pi^{-}$and $\mathbf{B}_{\mathrm{S}}^{\mathbf{0}} \rightarrow \mathbf{K}_{\mathrm{S}}^{\mathbf{0}} \mathbf{K}_{\mathrm{S}}^{\mathbf{0}}$}

Finally, we present our results for the $B_{s}^{0} \rightarrow K^{+} K^{-}, B_{s}^{0} \rightarrow K^{+} \pi^{-}, B_{s}^{0} \rightarrow \pi^{+} \pi^{-}$and $B_{s}^{0} \rightarrow K_{S}^{0} K_{S}^{0}$ charmless decays. The $B_{s}^{0} \rightarrow K^{+} K^{-}$mode is particularly interesting because it can be used for the determination of the CKM angle $\gamma$ [12] and may be sensitive to New Physics [13]. The charged pion and kaon candidates are selected using charged tracks and identified with energy deposition, momentum and time-of-flight measurements. The $K_{S}^{0}$ candidates are reconstructed via the $K_{S}^{0} \rightarrow$ $\pi^{+} \pi^{-}$decay, by selecting two oppositely-charged tracks matching various geometrical requirements [14]. A likelihood based on a Fisher discriminant using 16 modified Fox-Wolfram moments [15] is implemented to reduce the continuum, which is the main source of background.

We do observe a $5.8 \sigma$ excess of $24 \pm 6$ events in the $B_{s}^{*} \bar{B}_{s}^{*}$ region for the $B_{s}^{0} \rightarrow K^{+} K^{-}$mode (Fig. 3). The branching fraction $\mathscr{B}\left(B_{s}^{0} \rightarrow K^{+} K^{-}\right)=\left(3.8_{-0.9}^{+1.0}\right.$ (stat. $) \pm 0.7$ (syst. $\left.)\right) \times 10^{-5}$ is derived. On the other hand, no significant signal is seen for the other modes. Including the systematics uncertainties, we set the following upper limits at $90 \%$ confidence level: $\mathscr{B}\left(B_{s}^{0} \rightarrow \pi^{+} \pi^{-}\right)<$ 
$1.2 \times 10^{-5}, \mathscr{B}\left(B_{s}^{0} \rightarrow K^{+} \pi^{-}\right)<2.6 \times 10^{-5}$ and, assuming $\mathscr{B}\left(B_{s}^{0} \rightarrow K_{S}^{0} K_{S}^{0}\right)=\mathscr{B}\left(B_{s}^{0} \rightarrow K_{L}^{0} K_{L}^{0}\right)$, $\mathscr{B}\left(B_{s}^{0} \rightarrow K^{0} \bar{K}^{0}\right)<6.6 \times 10^{-5}$. The later is the first limit set for the $B_{s}^{0} \rightarrow K^{0} \bar{K}^{0}$ mode. All the other values are compatible with the CDF results $[16,17]$.
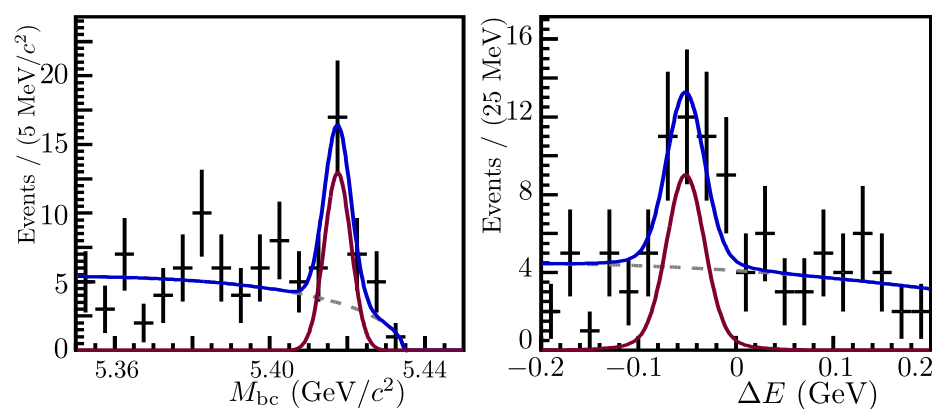

Figure 3: Projections of the $B_{s}^{0} \rightarrow K^{+} K^{-}$candidates (points with error bars) and the fitted PDF (solid blue line) in the $B_{s}^{*} \bar{B}_{s}^{*}$ signal region. The solid-red (dotted-grey) line represents the signal (continuum) component of the PDF.

\section{Conclusion}

All these studies demonstrate the great potential of the Belle data set recorded at $\Upsilon(5 S)$ energy. The sensitivity obtained for several $B_{s}^{0}$ modes allows many interesting studies. The branching fraction determinations of several important modes is ongoing and eight new measurements have been reported here. So far, the full Belle sample has reached $100 \mathrm{fb}^{-1}$, and the KEKB collider may continue delivering collisions at the $\Upsilon(5 S)$ energy during the fall 2009. Of course, many more interesting results are expected with the full Belle $\Upsilon(5 S)$ data set.

\section{References}

[1] A. Abashian et al. (Belle Collaboration), Nucl. Instrum. Methods A 479 (2002) 117.

[2] S. Kurokawa and E. Kikutani, Nucl. Instrum. Methods A 499 (2003) 1.

[3] A. Drutskoy et al. (Belle Collaboration), Phys. Rev. Lett. 98 (2007) 052001.

[4] A. Drutskoy et al. (Belle Collaboration), Phys. Rev. D 76 (2007) 012002.

[5] K. F. Chen et al. (Belle Collaboration), Phys. Rev. Lett. 100 (2008) 112001.

[6] G. S. Huang et al. (CLEO Collaboration), Phys. Rev. D 75 (2007) 012002.

[7] C. Amsler et al. (Particle Data Group), Phys. Lett. B 667 (2008) 1.

[8] R. Louvot et al. (Belle Collaboration), Phys. Rev. Lett. 102 (2009) 021801.

[9] G.C. Fox and S. Wolfram, Phys. Rev. Lett. 41 (1978) 1581.

[10] I. Dunietz, R. Fleischer and U. Nierste, Phys. Rev. D 63 (2001) 114015.

[11] A. Druskoy, ArXiv:0905.2959v1 [hep-ex] (2009).

[12] R. Fleischer, Phys. Lett. B 459 (1999) 306.

[13] D. London and J. Matias, Phys. Rev. D 70 (2004) 031502.

[14] F. Fang, Ph.D. thesis, University of Hawaii (2003).

[15] S.H. Lee et al. (Belle Collaboration), Phys. Rev. Lett. 91 (2003) 26.

[16] A. Abulencia et al. (CDF collaboration), Phys. Rev. Lett. 97 (2006) 211802.

[17] T. Aaltonen et al. (CDF collaboration), Phys. Rev. Lett. 103 (2009) 031801. 